\title{
Identification of host proteins interacting with Toxoplasma gondii GRA15 (TgGRA15) by yeast two-hybrid system
}

\author{
Qing Liu ${ }^{1,2}$ Fa-Cai Li $i^{*}$, Hany M. Elsheikha ${ }^{3}$, Miao-Miao Sun² and Xing-Quan Zhu',2,4
}

\begin{abstract}
Background: Toxoplasma gondii, an obligate intracellular protozoan parasite, possesses the remarkable ability to co-opt host cell machinery in order to maintain its intracellular survival. This parasite can modulate signaling pathways of its host through the secretion of polymorphic effector proteins localized in the rhoptry and dense granule organelles. One of such effectors is T. gondii type II-specific dense granule protein 15, TgGRA15, which activates NF-kB pathway. The aim of the present study was to identify the host interaction partner proteins of TgGRA15.

Methods: We screened a yeast two-hybrid mouse cDNA library using TgGRA15 as the bait. TgGRA15 (PRU strain, Type II) was cloned into the pGBKT7 vector and expressed in the Y2HGold yeast strain. Then, the bait protein expression was validated by western blotting analysis, followed by auto-activation and toxicity tests in comparison with control (Y2HGold yeast strain transformed with empty PGBKT7 vector).

Results: This screening led to the identification of mouse Luzp1 and AW209491 as host binding proteins that interact with TgGRA15. Luzp1 contains three nuclear localizing signals and is involved in regulating a subset of host non-coding RNA genes.

Conclusions: These findings reveal, for the first time, new host cell proteins interacting with TgGRA15. The identification of these cellular targets and the understanding of their contribution to the host-pathogen interaction may serve as the foundation for novel therapeutic and prevention strategies against T. gondii infection.
\end{abstract}

Keywords: Toxoplasma gondii, TgGRA15, Host-pathogen interaction, Yeast-two-hybrid, Luzp1, AW209491

\section{Background}

Toxoplasma gondii infection is a global public health challenge with approximately one third of the human population reported as chronic carriers [1]. This parasite infects and reproduces in virtually any nucleated cell of warm-blooded animals [2-4]. The disease that $T$. gondii causes in humans ranges from asymptomatic to debilitating or even fatal consequences in AIDS patients, organ transplants and during pregnancy $[5,6]$. In the last two decades, significant progress has been made towards a better understanding of the factors involved in the pathogenesis of $T$. gondii. These advances include

\footnotetext{
* Correspondence: lifacai@caas.cn

${ }^{2}$ State Key Laboratory of Veterinary Etiological Biology, Key Laboratory of Veterinary Parasitology of Gansu Province, Lanzhou Veterinary Research Institute, Chinese Academy of Agricultural Sciences, Lanzhou, Gansu Province 730046, People's Republic of China

Full list of author information is available at the end of the article
}

the identification of several parasite-derived effector proteins, such as ROPs and GRAs from the parasite rhoptry and dense granule organelles, respectively. These effectors are essential for $T$. gondii invasion and colonization [7-9]. For instance, GRAs direct the translocation into host cells of a battery of effector proteins, which modulate a variety of cellular processes including the maintenance of the parasitophorous vacuole (PV), gene expression, vesicle trafficking, and programmed cell death [7-14]. Modulation of these cellular processes allows $T$. gondii to replicate within host cells, avoid host defense mechanisms and ultimately sustain its intracellular survival.

About 30 of GRAs, such as TgGRA3, TgGRA5, TgGRA6, TgGRA7, TgGRA15, TgGRA16 and TgGRA18 have been identified [11, 15-18]. However, the exact roles of these proteins in the intracellular survival and 
growth of $T$. gondii are not completely understood. Of interest is the dense-granule protein GRA15 of Type II T. gondii strains, TgGRA15, which activates NF-kB signaling pathways most likely through activation of TRAF6, which in turn activates IкB kinase (IKK), leading to the phosphorylation and degradation of $I_{\kappa} B$, facilitating the subsequent translocation of $N F-k B$ to the nucleus to induce a broad range of genes involved in the immune response and inflammation [19, 20]. Also, TgGRA15 induces classical activation of macrophages $[10,11,13,21]$. These functions are critical in innate immune defense mechanism [13, 22-24]. TgGRA15 can also affect the accumulation of p65 guanylate binding protein 1 (GBP1) on the PV in the affected murine cells [25]. Even though TgGRA15 plays key roles in T. gondii pathogenesis, information of the interaction of TgGRA15 with TRAF6, IKK or murine GBP1 (mGBP1) protein remains hypothetical and the specific host cell interaction partner proteins of TgGRA15 are still unknown.

The advent of yeast-based screening assays, such as the yeast two-hybrid system [26, 27] has resulted in a considerable increase in the number of protein interactions reported in the scientific literature because these assays allow rapid discovery of new protein interactions via DNA library screening. In the yeast 2-hybrid $(\mathrm{Y} 2 \mathrm{H})$ assay, a given protein is assayed against a mixture of full-length proteins, protein domains and/or protein fragments expressed from a cDNA library, followed by isolation of the protein's interacting partners. What makes $\mathrm{Y} 2 \mathrm{H}$ assay a powerful tool for protein-interaction discovery is the lack of the need for prior knowledge of the interaction partners.

To advance the current understanding of the functions of TgGRA15, we attempted to identify host cellular proteins that interact with this parasite protein by means of the yeast two-hybrid system using mouse cDNA libraries. Results indicate that TgGRA15 interacts with Luzp1 and AW209491 host proteins. Identification of these two host proteins interacting with TgGRA15 may significantly improve the understanding of the role of TgGRA15 during T. gondii infection.

\section{Methods}

\section{Parasite strain}

Toxoplasma gondii (Type II) PRU strain was selected for generating a cDNA library because it encodes the TgGRA15, which allows type II strains of T. gondii to be a highly inducer of NF-kB activation, compared to type I strains [28] and type III strains [11]. The Type II PRU strain was maintained in our laboratory by the passage of cysts in female BALB/c mice (4 to 5 weeks of age). Mice were purchased from the Center of Experimental Animals, Lanzhou University, Lanzhou, China. Parasite maintenance in the mice experiment was performed in strict compliance with state and institutional animal care guidelines. The tissue cysts of $T$. gondii PRU strain were harvested from infected mice 30 days post-infection and were used for total RNA extraction.

\section{Bait plasmid construction}

Total RNA was extracted from parasite cysts by using TRIzol Reagent following the manufacturer's protocol (Invitrogen, Carlsbad, USA) and was reverse-transcribed into cDNA by using 1st strand cDNA Synthesis kit (TaKaRa, Dalian, China). To construct the TgGRA15 bait plasmid, the TgGRA15 gene fragment encoding a 352residue (from aa199 to aa550) peptide (Fig. 1a) was PCR amplified from the cDNA by using two gene-specific primers: TgGRA15-F (5'-GAATTCCCATCTACACTCA TACCTTCACCAG-3') and TgGRA15-R (5'-GGATCCT CATGGAGTTACCGCTGATTGTGTGTC-3'). The PCR product was ligated into pMD19-T vector (Takara, Dalian, China), which was then digested with restriction enzymes EcoRI and BamHI. The fragment was purified using a StarPrep Gel Extraction Kit (GenStar, Beijing, China), and then ligated into pGBKT7 vector at EcoR1 and $B a m \mathrm{H} 1$ sites. Subsequently, the plasmid (designated pGBKT7-TgGRA15) was transformed into E. coli DH5 $\alpha$ strain (Transgen, Beijing, China) and verified by endonuclease cleavage and sequencing (Sangon Biotech, Shanghai, China).

\section{TgGRA15 bait expression in yeast cells}

The pGBKT7-TgGRA15 bait plasmid was transformed into yeast strain Y2HGold using the Yeastmaker ${ }^{\mathrm{m}}$ Yeast Transformation System 2 kit (Cat. No. 630439, Clontech, Mountain View, USA). Then transformants were screened on an agar plate containing the minimal yeast medium without tryptophan (SD/-Trp) for 3-5 days. To check the expression of TgGRA15 bait in yeast cells, one colony from the SD/-Trp plate was picked to prepare a $5 \mathrm{ml}$ overnight culture, which was then inoculated into $50 \mathrm{ml}$ YPDA medium and incubated at $30{ }^{\circ} \mathrm{C}$ with shaking until the OD600 reached 0.4-0.6. The culture was centrifuged, and total proteins were extracted from the pelleted cells by the Urea/SDS method [29, 30]. Extracted proteins were then separated by $12 \%$ SDS-PAGE gels and transferred onto PVDF membrane (Millipore, Billerica, USA). The membrane was incubated with Myc-Tag Mouse mAb (CST, Danvers, USA) for $1 \mathrm{~h}$. Following three $10 \mathrm{~min}$ washings in TBST buffer, the membrane was incubated with HRPconjugated goat anti-mouse secondary antibody (Sigma, St. Louis, USA). The blot was observed using the direct ECL chemiluminescent method (GE, Buckinghamshire, USA). The yeast strain Y2HGold was transformed with pGBKT7-53 using the same method described above and was used as a positive control. 


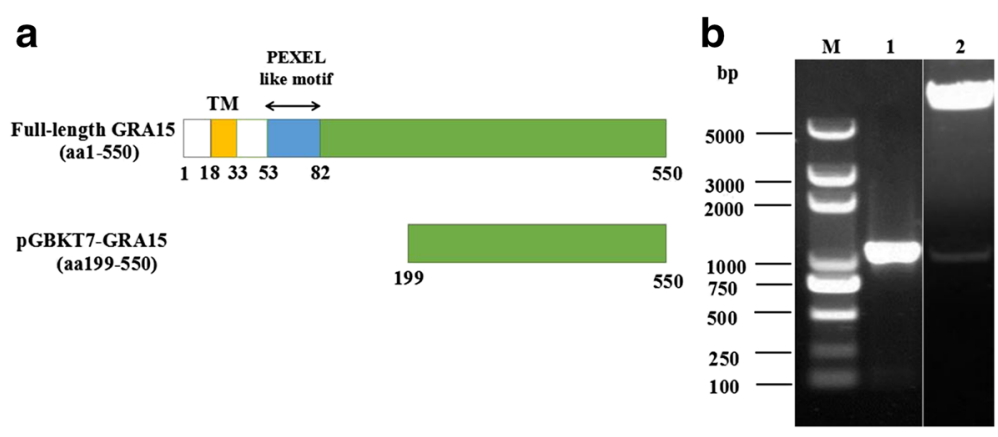

Fig. 1 Construction of the pGBKT7-TgGRA15 bait plasmid. a Schematic illustration of full-length TgGRA15 and the region of TgGRA15 used in the yeast-two-hybrid screen. $\mathbf{b}$ Lane M: DL5000 DNA Marker; Lane 1: the gel electrophoresis of the fragment of TgGRA15 amplified from T. gondii cDNA; Lane 2: the constructed pGBKT7-TgGRA15 bait plasmid was identified by digestion with EcoRl and BamHI

\section{Autoactivation and toxicity tests}

To test autoactivation of the bait protein, the pGBKT7TgGRA15 and the empty pGBKT7 plasmids were transformed into the yeast strain Y2HGold, respectively. Transformants were then grown on SD/-Trp, SD/-Trp/ $\mathrm{X}-\alpha-\mathrm{Gal}(40 \mu \mathrm{g} / \mathrm{ml} \mathrm{X}-\alpha-\mathrm{Gal})$ and SD/-Trp/X- $\alpha-\mathrm{Gal} / \mathrm{AbA}$ $(40 \mu \mathrm{g} / \mathrm{ml} \mathrm{X}-\alpha-\mathrm{Gal}$ and $125 \mathrm{ng} / \mathrm{ml}$ Aureobasidin A) agar plates for 3-5 days. The bait that did not autonomously activate the reporter gene was indicated by white colonies on SD/-Trp and SD/-Trp/X- $\alpha$-Gal plates, and the absence of colonies on SD/-Trp/X- $\alpha-G a l / A b A$ agar plates. Meanwhile, the colony size of Y2HGold transformed with bait plasmid was compared to Y2HGold transformed with the empty pGBKT7 vector. If the bait is toxic, colonies containing the bait vector will be smaller than colonies containing the empty pGBKT7 vector. Only the bait that lacks autoactivation and toxicity was used in the $\mathrm{Y} 2 \mathrm{H}$ screening.

\section{$\mathrm{Y} 2 \mathrm{H}$ screening using yeast mating}

All yeast strains and reagents for $\mathrm{Y} 2 \mathrm{H}$ assays were purchased from Clontech Co. (Mountain View, USA). To identify host cellular proteins that interact with TgGRA15, we screened this protein against a mouse cDNA library. Briefly, the Y187 cells harboring the Mate \& Plate $^{\mathrm{TM}}$ Universal Mouse (Normalized) cDNA library (Cat. No. 630482, Clontech, Mountain View, USA) cloned into the pGADT7-RecAB vector were used to mate with the Y2HGold cells transformed with pGBKT7-TgGRA15 for $20-24 \mathrm{~h}$ at $30{ }^{\circ} \mathrm{C}$. The mated culture was then spread onto SD/-Leu/-Trp/X- $\alpha-\mathrm{Gal} /$ AbA plates (DDO/X/A). Blue colonies were patched out onto higher stringency SD/-Ade/-His/-Leu/-Trp/X- $\alpha$ Gal/AbA (QDO/X/A) plates. To estimate each insert size on potential positive prey plasmids, PCR amplification was performed using Matchmaker ${ }^{\mathrm{TM}}$ AD LD-Insert Screening Amplimer Set (Cat. No. 630433, Clontech, Mountain View, USA).

\section{Rescuing the prey plasmids and confirmation of the interactions}

To rescue plasmids from yeast, the prey plasmids in putatively positive hits were isolated using the Easy Yeast Plasmid Isolation Kit (Cat. No. 630467, USA) and were purified by transformation of $E$. coli $\mathrm{DH} 5 \alpha$ competent cells (Transgen, Beijing, China), followed by selection on LB/Amp agar plates and plasmid isolation. To confirm the potential positive interactions, small-scale matings were performed as shown in Table 1 . Briefly, each potential positive prey plasmid from the initial screen was transformed into Y187 strain, followed by mating with Y2HGold yeast strain containing pGBKT7-TgGRA15 bait plasmid or empty pGBKT7 vector, respectively. The mated cultures were then spread onto QDO/X/A agar plates. Y2HGold transformed with pGBKT7-53 or pGBKT7 vector were used to mate with Y187 cells containing pGADT7-T, and were used as control.

\section{Positive prey analysis}

The isolated prey plasmids were sequenced by $\mathrm{T} 7$ primer and the sequences obtained were blasted against NCBI (National Center for Biotechnology Information) databases to analyze the function of the corresponding mouse genes. Also, based on Gene Ontology (GO) categories, gene functional classification of the identified genes was performed by searching the Mouse Genome Informatics database (http://www.informatics.jax.org/).

\section{Results}

\section{Construction of the bait plasmid}

The fragment of TgGRA15 was successfully amplified from cDNA of $T$. gondii PRU strain, as shown in Fig. 1b, Lane 1. The pGBKT7-TgGRA15 bait plasmid was constructed as described in Methods. The construction of the resultant plasmid was checked and confirmed by restriction enzyme digestion (Fig. 1b, Lane 2). 
Table 1 Reconfirmation of the potential positive interactions using small-scale mating

\begin{tabular}{|c|c|c|c|c|c|c|c|c|}
\hline \multirow[t]{2}{*}{ Y2HGold yeast strain } & \multicolumn{8}{|c|}{ Y187 yeast strain } \\
\hline & Prey1 & Prey2 & Prey3 & Prey4 & Prey5 & Prey6 & Prey7 & Prey8 \\
\hline Empty pGBKT7 vector & $\mathrm{C} 1$ & C2 & C3 & C4 & C5 & C6 & C7 & $\mathrm{C} 8$ \\
\hline pGBKT7-GRA15 bait plasmid & 1 & 2 & 3 & 4 & 5 & 6 & 7 & 8 \\
\hline
\end{tabular}

\section{Expression, auto-activation and toxicity tests of TgGRA15 bait in yeast cells}

Before $\mathrm{Y} 2 \mathrm{H}$ screening, the expression of the fusion protein was confirmed. Total proteins of the yeast strain Y2HGold transformed with pGBKT7-TgGRA15 bait plasmid were extracted and detected by western blotting using the c-Myc epitope tag antibody. The relative molecular weight of the GRA15 bait fusion recognized on the western blot was approximately $57 \mathrm{kDa}$, which is consistent with its estimated size (Fig. 2a, Lane 1). Y2HGold cells containing the pGBKT7-53 vector that expressed a $57 \mathrm{kDa}$ protein was used as a control (Fig. 2a, Lane 2).

To test the autoactivation activity of the bait protein in yeast cells, the pGBKT7 bait plasmid was transformed into Y2HGold cells, and subsequently the transformants were grown on SD/-Trp, SD/-Trp/X- $\alpha-G a l$ and SD/Trp/X- $\alpha-G a l / A b A$ (Fig. 2b). Autoactivation activities from the bait would enable the expression of reporter genes and result in blue colonies on $\mathrm{SD} /-\mathrm{Trp} / \mathrm{X}-\alpha-\mathrm{Gal}$ and $\mathrm{SD} /-\operatorname{Trp} / \mathrm{X}-\alpha-\mathrm{Gal} / \mathrm{AbA}$ plates. The results showed that no autoactivation activity was detected from TgGRA15, as shown in Fig. 2b. Furthermore, the colony size of Y2HGold transformed with bait plasmid was similar to that of Y2HGold transformed with the empty pGBKT7 vector. Based on these results, the pGBKT7TgGRA15 bait plasmid was used in the $\mathrm{Y} 2 \mathrm{H}$ screening.

\section{$\mathrm{Y} 2 \mathrm{H}$ screening against a mouse cDNA library using TgGRA15 as bait}

To screen host proteins that interplay with the GRA15 bait by $\mathrm{Y} 2 \mathrm{H}$ system, Y2HGold cells containing the pGBKT7-TgGRA15 plasmid were used to mate with Y187 cells harboring the Mate \& Plate $^{\mathrm{TM}}$ Universal Mouse (Normalized) cDNA library. Based on the number of colonies on different selection plates, the mating efficiency was calculated to be $6.8 \%$ and $5 \times 10^{6}$ colonies were screened. Among 72 colonies $(2-3 \mathrm{~mm})$ grown on DDO/X/A plates, 18 were blue. Subsequently, these 18 blue clones were patched out onto higher stringency QDO/X/A agar plates. As a result, eight out of the 18 colonies still turned blue. The prey plasmids were then isolated from these potential positive interactors and rescued by transformation into $E$. coli $\mathrm{DH} 5 \alpha$ competent cells. The specific insert on each prey plasmid was PCR amplified using T7 sequencing primer and 3'AD sequencing primer. The results of PCR amplification are shown in Fig. 3a. To confirm the specificity of interaction, Y187 strain transformed with each potential positive prey plasmid (preys 1-8) was used to mate with Y2HGold containing pGBKT7-TgGRA15 bait plasmid. The results showed that all of the zygotes resulted from mating turned blue on QDO/X/A plates (Fig. 3b, 1-8). To eliminate the false positive hits, Y187 strain transformed with
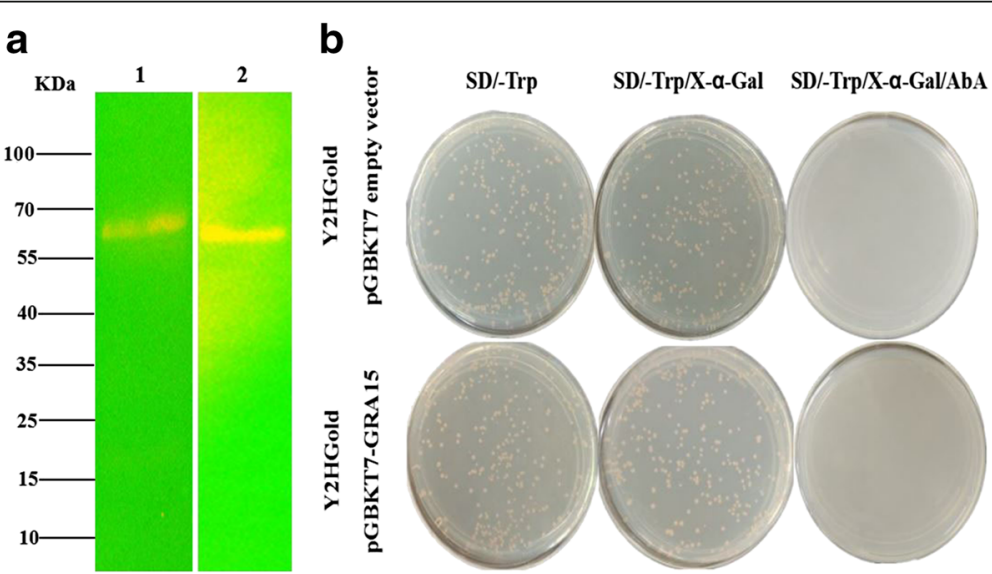

Fig. 2 Expression, auto-activation and toxicity tests for the TgGRA15 bait. a Western blotting analysis of total protein extracts of Y2HGold containing the following plasmids: Lane 1: pGBKT7-TgGRA15; Lane 2: pGBKT7-53. b Determination of the auto-activation and toxicity activity of the pGBKT7-TgGRA15 bait plasmid in yeast cells. The pGBKT7-TgGRA15 bait plasmid and empty pGBKT7 vector were used to transform Y2HGold cells and then grown on different plates 


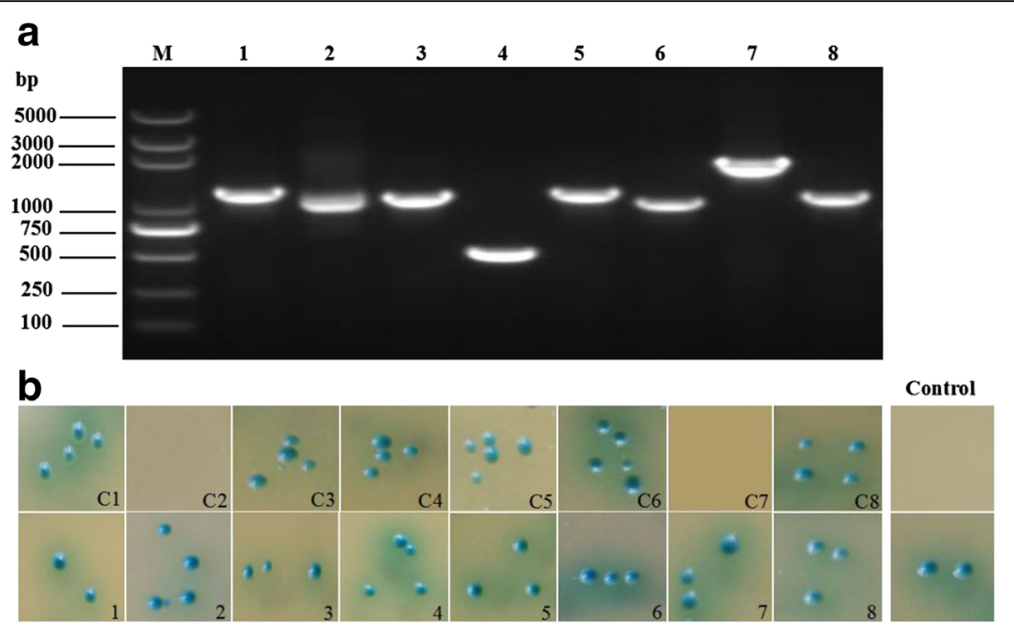

Fig. 3 Analysis of putatively positive clones. a Gel electrophoresis of PCR products amplified from putatively positive prey plasmids. Lane M: DL5000 DNA Marker; Lanes 1-8: eight blue clones containing prey1-8 (prey plasmid isolated from eight blue clones), respectively, were amplified by $T 7$ sequencing primer and 3'AD sequencing primer. b Confirmation of putatively positive clones; C1-C8: zygotes formed from Y187 cells containing Prey1-8 mating with Y2HGold containing empty pGBKT7 vector, respectively. 1-8: zygotes formed from Y187 cells containing Prey1-8 mating with Y2HGold containing pGBKT7GRA15 bait plasmid, respectively. Control, Y2HGold transformed with pGBKT7-53 or empty pGBKT7 vector were used to mate with Y187 cells containing pGADT7-T, respectively. The mated cultures were then grown on QDO/X/A agar plates, respectively

each potential positive prey plasmid (preys 1-8) was then used to mate with Y2HGold containing empty pGBKT7 vector. In this case, C2 and C7 showed no colony on $\mathrm{QDO} / \mathrm{X} / \mathrm{A}$ plates, whereas the others still turned blue (Fig. 3b). The control of Y187 containing pGADT7-T mated with Y2HGold containing pGBKT7-53 showed blue colonies, whereas mating with Y2HGold containing empty pGBKT7 vector showed no colony on QDO/X/A plates. Compared to the results of the control, two host proteins were identified to interact with TgGRA15.

\section{Sequencing and analysis of positive prey}

To determine the identity of positive clones, the two prey plasmids were sequenced using T7 primer. The sequencing results were analyzed by Basic Local Alignment Search Tool (BLAST) in NCBI, which revealed that the two inserts had $100 \%$ sequence identity to that of the two Mus musculus genes: the expressed sequence AW209491 (GenBank accession nos. AW209491 and NM_134067.4) and the leucine zipper protein 1 (Luzp1, GenBank accession no. BC053451.1). The sequencing results also showed that the AW209491 containing hit encoded a 100-residue fragment fused to the GAL4 DNA-binding domain, and the Luzp1 containing hit encoded a 104-residue region. These two proteins were not previously reported to interact with TgGRA15. Luzp1 is an annotated protein while AW209491 is of unknown function. The biological process analysis of Luzp1 revealed that Luzp1 is related to system development (artery development, neural fold bending and ventricular septum development) in the biological process.
Also, in the cellular component ontology, Luzp1 was related to extracellular region and nucleus.

\section{Discussion}

TgGRA15 is involved in several critical functions, including activation of the NF- $\mathrm{kB}$ signaling pathway. Despite its importance, information about the host binding partners of TgGRA15 is lacking. To our knowledge, this report reveals for the first time two host proteins that interact with TgGRA15. The study was focused on TgGRA15 of Type II (not Type I and Type III) because Type II is more efficient in the activation of NF- $\mathrm{kB}$, an important host signaling pathway that regulates inflammatory, immune and antiapoptotic responses [11].

Structure analysis of TgGRA15 showed that it contains a transmembrane domain and a PEXEL-like motif (Fig. 1a), a sorting signal that directs Plasmodium proteins into erythrocytes, whereas the $T$. gondii PEXELlike motif contributes to PVM association [31]. In the present study, the 1,056 bp fragment encoding a 352residue (from aa199 to aa550) peptide of TgGRA15 without the transmembrane domain and the PEXEL-like motif was cloned into pGBKT7 vector as the bait plasmid, followed by screening the host cell molecules that interact with TgGRA15 Type II by $\mathrm{Y} 2 \mathrm{H}$ assay. Our results revealed two mouse proteins, AW209491 and Luzp1 that interact with TgGRA15.

Previous studies demonstrated that Luzp, a leucine zipper motif-containing protein, harbors three nuclear localizing signals and numerous putative Ser/Thr phosphorylation sites [32]. Luzp is mainly expressed in the brain, and the knockout of Luzp can lead to failure of 
cranial neural tube closure, indicating that Luzp plays an important role in the development of embryonic brain [32, 33]. Although there are three proteins, Luzp1, Luzp2 and Luzp4 in mice, the "Luzp" aforementioned is mapped to mouse chromosome 4 [32], which is consistent with the distribution of Luzp1, but not Luzp2 and Luzp4, which were mapped to Chromosome 7 and Chromosome $\mathrm{X}$, respectively. Luzp1 can serve as a bridge factor between the AdaTwo-A-containing histone acetyltransferase (ATAC) and the Mediator complex (MED), and as a result a highly stable meta-coactivator complex (MECO) formed, which is a regulator of a subset of non-coding RNA genes [34].

GRA15 was reported to be detected at the PV, as well as host cell cytoplasm and host cell nucleus $[11,35]$, however, there is no evidence for how GRA15 targets the host cell nucleus. In addition, structure analysis of GRA15 showed no nuclear localization signal [35]. Given that Luzp1 interacts with TgGRA15, we speculate that TgGRA15 may target the host cell nucleus by interacting with Luzp1. This finding provides a basis for how TgGRA15 translocates to host cell nucleus.

As yeast is a eukaryote cell, proteins encoded by yeast expression system are likely to be similar to their natively expressed ones. Therefore, $\mathrm{Y} 2 \mathrm{H}$ system can be an effective and accurate method for large-scale screening of candidate proteins interacting with the bait, and in our study, we have identified two host proteins interacting with TgGRA15. There are probably other host proteins that also interact with TgGRA15, but were not detected in our screening, probably because the Universal Mouse (Normalized) cDNA library may not contain the clones that express potential interactors in the correct manner, due to a reading-frame shift. In addition, as the bait used is a 352-residue, the potential interactors may interact with other regions of TgGRA15. It is also possible, that some host proteins were not identified either due to the host protein not being present in the library or due to the inability of the protein to interact due to the limitations of the $\mathrm{Y} 2 \mathrm{H}$ system. Further work is needed in order to evaluate the importance of the interactions described here with regards to their possible roles in $T$. gondii replication or virulence, as our study was limited to identification of proteins in the $\mathrm{Y} 2 \mathrm{H}$, and there is the possibility that some of these protein interactions are not occurring in cells that are infected with the parasite. Nevertheless, the identification of two cellular factors potentially interacting with TgGRA15 during $T$. gondii infection is critical to elucidate processes known to involve TgGRA15 as well as to provide some new clues for studying the biological function of TgGRA15.

\section{Conclusions}

In conclusion, we identified two mouse proteins, Luzp1 and AW209491, that interact with TgGRA15. Luzp1 is a nucleus-targeted protein which is related to system development in the biological process ontology and involved in the regulation of a subset of host non-coding RNA genes. Due to the limitations of the $\mathrm{Y} 2 \mathrm{H}$ system, the proteins identified are not exhaustive and interactions identified need to be confirmed by independent experimental approaches in the context of $T$. gondiiinfected cells before making any definitive conclusion on the relevance for the parasite life-cycle. Understanding the specific mechanisms underlying TgGRA15 interaction with host partner proteins and the effect of this interaction on the regulation of the NF-kB signaling may lead to the identification of novel therapeutic strategies for the prevention and treatment of $T$. gondii infection.

\section{Abbreviations \\ AIDS: Acquired immunodeficiency syndrome; BLAST: Basic Local Alignment Search Tool; cDNA: complementary deoxyribonucleic acid; GBP1: guanylate binding protein 1; GO: Gene Ontology; IKK: IKB kinase; Luzp1: Leucine zipper protein 1; NCBI: National Center for Biotechnology Information; \\ NF-kB: Nuclear factor kappa B; PCR: Polymerase chain reaction; PRU: Prugniaud; PV: Parasitophorous vacuole; ROPs: Rhoptry proteins; TgGRA15: T. gondii dense granule protein 15; $\mathrm{Y} 2 \mathrm{H}$ : Yeast 2-hybrid}

\section{Acknowledgements}

Not applicable.

\section{Funding}

Project support was provided by the National Natural Science Foundation of China (Grant No. 31230073).

\section{Availability of data and materials}

Not applicable.

\section{Authors' contributions}

XQZ and FCL conceived and designed the study, and critically revised the manuscript. QL performed all of the experiments and drafted the manuscript. MMS helped carry out yeast-two-hybrid screening. HME contributed to the revision of the manuscript. All authors read and approved the final manuscript.

\section{Competing interests}

The authors declare that they have no competing interests.

Consent for publication

Not applicable.

\section{Ethics approval}

The study design was reviewed and approved by the Animal Ethics Committee of Lanzhou Veterinary Research Institute, Chinese Academy of Agricultural Sciences.

\footnotetext{
Author details

${ }^{1}$ College of Veterinary Medicine, Hunan Agricultural University, Changsha, Hunan Province 410128, People's Republic of China. 'State Key Laboratory of Veterinary Etiological Biology, Key Laboratory of Veterinary Parasitology of Gansu Province, Lanzhou Veterinary Research Institute, Chinese Academy of Agricultural Sciences, Lanzhou, Gansu Province 730046, People's Republic of China. ${ }^{3}$ Faculty of Medicine and Health Sciences, School of Veterinary Medicine and Science, University of Nottingham, Sutton Bonington Campus, Loughborough LE12 5RD, UK. ${ }^{4}$ Jiangsu Co-innovation Center for Prevention and Control of Important Animal Infectious Diseases and Zoonoses, Yangzhou, Jiangsu Province 225009, People's Republic of China.
} 
Received: 29 October 2016 Accepted: 15 December 2016 Published online: 03 January 2017

\section{References}

1. Montoya JG, Liesenfeld O. Toxoplasmosis. Lancet. 2004;363:1965-76.

2. Dubremetz JF. Host cell invasion by Toxoplasma gondii. Trends Microbiol. 1998;6:27-30.

3. Dubey JP. Toxoplasmosis of Animals and Humans. 2nd ed. Boca Raton: CRC Press; 2010. p. 313

4. Liu Q, Wang ZD, Huang SY, Zhu XQ. Diagnosis of toxoplasmosis and typing of Toxoplasma gondii. Parasit Vectors. 2015;8:292.

5. Elsheikha HM. Congenital toxoplasmosis: priorities for further health promotion action. Public Health. 2008:122:335-53.

6. Minbaeva G, Schweiger A, Bodosheva A, Kuttubaev O, Hehl AB, Tanner I, et al. Toxoplasma gondii infection in Kyrgyzstan: seroprevalence, risk factor analysis, and estimate of congenital and AIDS related toxoplasmosis. PLOS Negl Trop Dis. 2013;7:e2043.

7. Bougdour A, Durandau E, Brenier-Pinchart MP, Ortet P, Barakat M, Kieffer S, et al. Host cell subversion by Toxoplasma GRA16, an exported dense granule protein that targets the host cell nucleus and alters gene expression. Cell Host Microbe. 2013;13:489-500.

8. Braun L, Brenier-Pinchart MP, Yogavel M, Curt-Varesano A, Curt-Bertini RL, Hussain T, et al. A Toxoplasma dense granule protein, GRA24, modulates the early immune response to infection by promoting a direct and sustained host p38 MAPK activation. J Exp Med. 2013;210:2071-86.

9. Jensen KD, Hu K, Whitmarsh RJ, Hassan MA, Julien L, Lu D, et al. Toxoplasma rhoptry kinase ROP16 promotes host resistance to oral infection and intestinal inflammation only in the context of the dense granule protein GRA15. Infect Immun. 2013;81:2156-67.

10. Jensen KD, Wang Y, Wojno ED, Shastri AJ, Hu K, Cornel L, et al. Toxoplasma polymorphic effectors determine macrophage polarization and intestinal inflammation. Cell Host Microbe. 2011;9:472-83.

11. Rosowski EE, Lu D, Julien $L$, Rodda L, Gaiser RA, Jensen KD, et al. Strainspecific activation of the NF-kappaB pathway by GRA15, a novel Toxoplasma gondii dense granule protein. J Exp Med. 2011;208:195-212.

12. Niedelman W, Gold DA, Rosowski EE, Sprokholt JK, Lim D, Farid Arenas A, et al. The rhoptry proteins ROP18 and ROP5 mediate Toxoplasma gondii evasion of the murine, but not the human, interferon-gamma response. PLoS Pathog. 2012;8:e1002784.

13. Gov L, Karimzadeh A, Ueno N, Lodoen MB. Human innate immunity to Toxoplasma gondii is mediated by host caspase- 1 and ASC and parasite GRA15. MBio. 2013;4:e00255-13.

14. Gold DA, Kaplan AD, Lis A, Bett GC, Rosowski EE, Cirelli KM, et al. The Toxoplasma Dense Granule Proteins GRA17 and GRA23 mediate the movement of small molecules between the host and the parasitophorous vacuole. Cell Host Microbe. 2015;17:642-52

15. Jacobs D, Vercammen M, Saman E. Evaluation of recombinant dense granule antigen 7 (GRA7) of Toxoplasma gondii for detection of immunoglobulin G antibodies and analysis of a major antigenic domain. Clin Diagn Lab Immunol. 1999:6:24-9.

16. Fazaeli A, Carter PE, Darde ML, Pennington TH. Molecular typing of Toxoplasma gondii strains by GRA6 gene sequence analysis. Int J Parasitol. 2000;30:637-42

17. Kong JT, Grigg ME, Uyetake L, Parmley SF, Boothroyd JC. Serotyping of Toxoplasma gondii infections in humans using synthetic peptides. J Infect Dis. 2003;187:1484-95.

18. Chen J, Li ZY, Zhou DH, Liu GH, Zhu XQ. Genetic diversity among Toxoplasma gondii strains from different hosts and geographical regions revealed by sequence analysis of GRA5 gene. Parasit Vectors. 2012;5:279.

19. DiDonato JA, Mercurio F, Karin M. NF-KB and the link between inflammation and cancer. Immunol Rev. 2012;246:379-400.

20. Gilmore TD, Wolenski FS. NF-kappaB: where did it come from and why? Immunol Rev. 2012;246:14-35.

21. Xie $Y$, Wen $H$, Yan $K$, Wang $S$, Wang $X$, Chen J, et al. Toxoplasma gondii GRA15II effector-induced M1 cells ameliorate liver fibrosis in mice infected with Schistosomiasis japonica. Cell Mol Immunol. 2016;Epub ahead of print.

22. Sibley LD. Invasion and intracellular survival by protozoan parasites. Immunol Rev. 2011;240:72-91.

23. Walker DM, Oghumu S, Gupta G, McGwire BS, Drew ME, Satoskar AR. Mechanisms of cellular invasion by intracellular parasites. Cell Mol Life Sci. 2014;71:1245-63.
24. Hakimi MA, Bougdour A. Toxoplasma's ways of manipulating the host transcriptome via secreted effectors. Curr Opin Microbio. 2015;26:24-31.

25. Virreira Winter S, Niedelman W, Jensen KD, Rosowski EE, Julien L, Spooner E, et al. Determinants of GBP recruitment to Toxoplasma gondii vacuoles and the parasitic factors that control it. PLoS One. 2011;6:e24434.

26. Fields S, Sternglanz R. The two-hybrid system: an assay for protein-protein interactions. Trends Genet. 1994;10:286-92.

27. Miller J, Stagljar I. Using the yeast two-hybrid system to identify interacting proteins. Methods Mol Biol. 2004;261:247-62.

28. Robben PM, Mordue DG, Truscott SM, Takeda K, Akira S, Sibley LD. Production of IL-12 by macrophages infected with Toxoplasma gondii depends on the parasite genotype. J Immunol. 2004;172:3686-94.

29. Printen JA, Sprague Jr GF. Protein-protein interactions in the yeast pheromone response pathway: Ste5p interacts with all members of the MAP kinase cascade. Genetics. 1994;138:609-19.

30. Wang Y, Fang R, Yuan Y, Hu M, Zhou Y, Zhao J. Identification of host proteins interacting with the integrin-like A domain of Toxoplasma gondii micronemal protein MIC2 by yeast-two-hybrid screening. Parasit Vectors. 2014;7:543.

31. Hsiao CH, Luisa Hiller N, Haldar K, Knoll LJ. A HT/PEXEL motif in Toxoplasma dense granule proteins is a signal for protein cleavage but not export into the host cell. Traffic. 2013;14:519-31.

32. Sun DS, Chang AC, Jenkins NA, Gilbert DJ, Copeland NG, Chang NC. Identification, molecular characterization, and chromosomal localization of the CDNA encoding a novel leucine zipper motif-containing protein. Genomics. 1996:36:54-62.

33. Hsu CY, Chang NC, Lee MW, Lee KH, Sun DS, Lai C, et al. LUZP deficiency affects neural tube closure during brain development. Biochem Biophys Res Commun. 2008:376:466-71.

34. Krebs AR, Demmers J, Karmodiya K, Chang NC, Chang AC, Tora L. ATAC and Mediator coactivators form a stable complex and regulate a set of non-coding RNA genes. EMBO Rep. 2010;11:541-7.

35. Mercier C. Cesbron-Delauw. MF Toxoplasma secretory granules: one population or more? Trends Parasitol. 2015;31:60-71.

\section{Submit your next manuscript to BioMed Central and we will help you at every step:}

- We accept pre-submission inquiries

- Our selector tool helps you to find the most relevant journal

- We provide round the clock customer support

- Convenient online submission

- Thorough peer review

- Inclusion in PubMed and all major indexing services

- Maximum visibility for your research

Submit your manuscript at www.biomedcentral.com/submit
) Biomed Central 